\title{
The Three Page Guide to the Most Important Results of M. S. El Naschie's Research in E-Infinity Quantum Physics and Cosmology
}

\author{
M. A. Helal ${ }^{1}$, L. Marek-Crnjac ${ }^{2}$, Ji-Huan $\mathrm{He}^{3}$ \\ ${ }^{1}$ Department of Math, Cairo University, Egypt \\ ${ }^{2}$ Technical School Center of Maribor, Maribor, Slovenia \\ ${ }^{3}$ National Engineering Laboratory for Modern Silk, \\ College of Textile and Clothing Engineering, Soochow University, Suzhou, China \\ Email: leila.marek@guest.arnes.si, hejihuan@suda.edu.cn
}

Received October 28, 2013; revised November 10, 2013; accepted November 17, 2013

Copyright (C) 2013 M. A. Helal et al. This is an open access article distributed under the Creative Commons Attribution License, which permits unrestricted use, distribution, and reproduction in any medium, provided the original work is properly cited.

\begin{abstract}
In this short survey, we give a complete list of the most important results obtained by El Naschie's E-infinity Cantorian space-time theory in the realm of quantum physics and cosmology. Special attention is paid to his recent result on dark energy and revising Einstein's famous formula $E=m c^{2}$.
\end{abstract}

Keywords: Review of E-infinity; Summary of Cantorian Space-Time; El Naschie Nottale and Ord Fractal Space-Time; Rindler Space-Time; Revising Einstein Theory; Dark Energy Revealed

\section{Introduction}

The present letter could have been entitled a short history of space-time. It is mainly concerned with space, time and matter and gives an almost exhaustive list of the most important results obtained by El Naschie using his Cantorian-fractal theory of space-time [1-85].

In a 2012 conference in the Bibliotheca Alexandrina [84] $E_{1}$ Naschie announced the revision of Einstein's special relativity energy-mass formula $E=m c^{2}$ to $E=m c^{2} / 22$ and showed that it is the sum of the energy of the quantum particle $E_{1}=m c^{2} / 22$ and the energy of the quantum wave $E_{2}=m c^{2}$ (21/22) [53-63]. He also showed that the speed of light is an exact expectation value in classical three plus one dimensions.

\section{Guide to the Most Important Results of E-Infinity Theory in Physics and Astrophysics}

El Naschie's E-Infinity theory $E^{\infty}$ has clearly shown that random Cantor sets are the basic building blocks of quantum space-time. The intrinsic topological dimension of these building blocks is zero; its Hausdorff fractal dimension is the inverse of golden ratio $(0.618033989 . .$. and its embedding dimension is unity $[21,24,25]$.

El Naschie made it plausible that because orthodox quantum mechanics is totally insensitive to fractals and does not consider the Cantorian nature of quantum spacetime, many paradoxes following $[1,4,71]$.

El Naschie proved that quantum space-time is described fully by not one but three dimensions. First, a topological (Menger-Urysohn) dimension equals to exactly 4. Second, a Hausdorff dimension equals to 4 plus the inverse of the golden ratio to the power of 3 which means $4.236067977 \ldots$ This is effectively a $4 D$ cube inside a $4 D$ cube and so on ad infinitum. Third, a formal dimension equals to infinity. In other words, our quantum spacetime is an infinitely dimensional but hierarchal Cantor set of measure zero [64].

Many of the fundamental constants of nature were derived by El Naschie from first principles. This includes the coupling constant of quantum gravity as well as the electromagnetic fine structure constant and Newton's gravity constant. In particular a fundamental equation was established relating the Bulk (E8E8) with the holographic boundary and gravity from which $\bar{\alpha}=137$ was derived [4,15,23,35,45].

An exact renormalization equation was constructed 
which derived quarks confinement as an exact result [4143].

The relationship between Lie symmetry groups as well as two and three Stein spaces and high energy physics was outlined. In particular the role played by $E 8$ in this respect was analyzed. The total number of elementary particles in an extended standard model was shown to be 137 particles. This includes 10 space-time quasi dimensions. E-infinity P-Adic reasoning was also employed $[16,44,45]$.

The stationary state of quantum mechanics was shown to be that of the VAK, i.e. the vague attractor of Kolmogorov [78].

The theory predicted the existence of 8 dimensional Higgs field with at least one Higgs particle or five Higgs particles becoming manifest. The Higgs mass was determined to be approximately $169 \mathrm{GeV}$ [6].

The Euler characteristic as well as the curvature of the quantum space-time was shown to be equal to $26+k \cong$ 26. In addition, arguments were given to show that our universe is likely to be compact. An alternative theory using instanton density to calculate the first massless particle-like states corrected the classical well-known solution of Heterotic string theory, namely 8064 to the exact solution 8872 [25].

It is reasoned that in a totally disjointed infinitely dimensional but hierarchal Cantor-like space time manifold, calculus must be replaced by Weyl-like golden mean scaling which effectively represents a new version of quantized calculus $[25,38,56]$.

El Naschie made several suggestions regarding a $\mathrm{Ba}-$ nach-Tarski-like big bang theory based on paradoxical decomposition of spheres [51].

Mohamed El Naschie and his group proved the equivalent of his basic equations of E-infinity theory and the dimensional function of von Neumann's continuous geometry and A. Connes' non-commutative geometry $[33,34,49]$.

A quantum particle may be modelled as a fractal point is represented by the two dimensions of a zero set $\operatorname{dim} P$ $(0, \phi)$ where $\phi=(\sqrt{5}-1) / 2$. The quantum wave is the surface of a fractal point, i.e. a fractal surface and may be represented by the two dimensions of an empty set $\mathrm{dim}$ $W \equiv\left(-1, \phi^{2}\right)$. The empty set accounts for the paradoxical outcome of the two-slit experiment and the disappearance of the interference fringes [26].

It explained quantum entanglement as a zero measure fractal geometry [60].

It showed that dark energy is the negative energy of the quantum wave $E_{2}=E(D)=m c^{2}(21 / 22)$ which agrees completely with measurements [54-68].

It explained the meaning of the Immirzi parameter and Unruh temperature [53,54].

In agreement with the work of Magueijo and Smolin
[70], El Naschie showed that the speed of light is not constant but a constant expectation value [58].

Making a distinction between temporal chaos as an initial value problem and spatial chaos as a boundary value problem helped to arrive at fractal space-time, KAM space-time, the importance of the empty set, negative dimensions, Finkelstein quantum sets, Gödel theorem and the reality of the wave function $[8,9,18-20,71$, 72].

It derived the density of dark energy Nash embedding of Witten's fractal M-Theory [85].

\section{REFERENCES}

[1] M. S. El Naschie, O. E. Rössler and I. Prigogine, "Quantum Mechanics, Diffusion and Chaotic Fractals,” Elsevier Science Ltd., Oxford, 1995.

[2] M. Jammer, “Concepts of Space,” Dover Publications, New York, 1969.

[3] B. G. Sidharth, "The Universe of Fluctuations (The Architecture of Space-Time and the Universe)," Springer, Dordrecht, 2005.

[4] M. S. El Naschie, "Deterministic Quantum Mechanics versus Classical Mechanical Indeterminism,” International Journal of Nonlinear Science \& Numerical Simulation, Vol. 8, No. 1, 2007, pp. 5-10.

[5] M. S. El Naschie, "Average Exceptional Lie Group Hierarchy and High Energy Physics,” American Inst. of Physics, $9^{\text {th }}$ Int. Symposium Proceedings, 7-9 June 2008, AIP Conferences, 101018, pp. 15-20.

[6] J.-H. He, "Transfinite Physics: A Collection of Publication on E-Infinity Cantorian Space-Time Theory,” China Education and Culture Publishing Co., Beijing, 2005.

[7] J.-H. He, E. Goldfain, L. D. Sigalotti and A. Mejias, "Beyond the 2006 Physics Nobel Price for COBE: An Introduction to E-infinity Theory," China Education and Culture Publishing Co., Beijing, 2006.

[8] J. Brindly, T. Kapitaniak and M. S. El Naschie, “Analytical Conditions for Strange Chaotic and Non-Chaotic Attractors of the Quasi Periodically Forced van der Pol Equation,” Physica D: Nonlinear Phenomena, Vol. 51, No. 1-3, 1991, pp. 28-38. http://dx.doi.org/10.1016/0167-2789(91)90219-Y

[9] M. S. El Naschie and S. Al Athel, "On the Connection between Statical and Dynamical Chaos," Zeitschrift fur Naturforshung, Vol. 44a, 1989, pp. 645-650.

[10] D. K. Campbell, “Chaos,” AIP, New York, 1990.

[11] G. Cherbit, “Fractals,” J. Wiley, Chichester, 1991.

[12] S. Weinberg, “The Quantum Theory of Fields,” Parts I, II, III, Cambridge Press, 1998,2000.

[13] M. S. El Naschie, "Quantum Golden Field Theory-Ten theorems and Various Conjectures," Chaos, Solitons \& Fractals, Vol. 36, No. 5, 2008, pp. 1121-1125. http://dx.doi.org/10.1016/j.chaos.2007.09.023

[14] M. S. El Naschie, “Towards a Quantum Golden Field Theory,” International Journal of Nonlinear Sciences and 
Numerical Simulation, Vol. 8, No. 4, 2007, pp. 477-482.

[15] M. S. El Naschie, "High Energy Physics and the Standard Model from the Exceptional Lie Groups," Chaos, Solitons \& Fractals, Vol. 36, No. 1, 2008, pp. 1-17. http://dx.doi.org/10.1016/j.chaos.2007.08.058

[16] M. S. El Naschie, "P-Adic Unification of the Fundamental Forces and the Standard Model," Chaos, Solitons \& Fractals, Vol. 38, No. 4, 2008, pp. 1011-1012. http://dx.doi.org/10.1016/j.chaos.2008.04.047

[17] M. S. El Naschie, “On a Canonical Equation for All Fundamental Interactions,” Chaos, Solitons \& Fractals, Vol. 36, No. 5, 2008, pp. 1200-1204.

http://dx.doi.org/10.1016/j.chaos.2007.09.039

[18] M. S. El Naschie, "Statistical Mechanics of Multi-Dimensional Cantor Sets Gödel Theorem and Quantum SpaceTime,” Journal of Franklin Institute, Vol. 33, No. 1, 1993, pp. 199-211. http://dx.doi.org/10.1016/0016-0032(93)90030-X

[19] M. S. El Naschie, "Complex Dynamics in 4D PeanoHilbert Space,” Il Nuovo Cimento, Vol. 1, No. 5, 1992, pp. 583-594.

[20] M. S. El Naschie, "Peano Dynamics as a Model for Turbulence and Strange Non-Chaotic Behavior," Acta Physica Polonica A, Vol. 80, No. 1, 1991.

[21] M. S. El Naschie, "Quantum Mechanics and the Possibility of a Cantorian Space-Time," Chaos, Solitons \& Fractals, Vol. 1, No. 5, 1991, pp. 485-487. http://dx.doi.org/10.1016/0960-0779(91)90019-6

[22] M. S. El Naschie, "Renormalization Semi-Groups and the Dimension of Cantorian Space-Time," Chaos, Solitons \& Fractals, Vol. 4, No. 7, 1994, pp. 1141-1145. http://dx.doi.org/10.1016/0960-0779(94)90027-2

[23] M. S. El Naschie, "On a Class of General Theories for High Energy Particle Physics,” Chaos, Solitons \& Fractals, Vol. 14, No. 4, 2002, pp. 649-668.

http://dx.doi.org/10.1016/S0960-0779(02)00033-4

[24] M. S. El Naschie, "A Review of E-Infinity Theory and the Mass Spectrum of High Energy Particle Physics," Chaos, Solitons \& Fractals, Vol. 19, No. 1, 2004, pp. 209-236. http://dx.doi.org/10.1016/S0960-0779(03)00278-9

[25] M. S. El Naschie, "The Theory of Cantorian Space-Time and High Energy Particle Physics (An Informal Review)," Chaos, Solitons \& Fractals, Vol. 41, No. 5, 2009, pp. 2635-2646. http://dx.doi.org/10.1016/j.chaos.2008.09.059

[26] M. S. El Naschie, “Application of Chaos and Fractals in Fundamental Physics and Set Theoretical Resolution of the Two-Slit Experiment and Wave Collapse," The 3rd International Symposium on Nonlinear Dynamics, Donghua University, China, 2010, pp. 7-8.

[27] M. S. El Naschie, "Kaluza-Klein Unification-Some Possible Extensions," Chaos, Solitons \& Fractals, Vol. 37, No. 1, 2008, pp. 16-22.

http://dx.doi.org/10.1016/j.chaos.2007.09.079

[28] M. S. El Naschie, "On Dualities between Nordstrom-Kaluza-Klein Newtonian and Quantum Gravity," Chaos, Solitons \& Fractals, Vol. 36, No. 4, 2008, pp. 808-810. http://dx.doi.org/10.1016/j.chaos.2007.09.019
[29] M. S. El Naschie, "Superstring Theory: What It Cannot Do but E-Infinity Could," Chaos, Solitons \& Fractals, Vol. 29, No. 1, 2006, pp. 65-68. http://dx.doi.org/10.1016/j.chaos.2005.11.021

[30] G. N. Ord, “Fractal Space-Time,” Journal of Physics A: Mathematical and General, Vol. 16, No. 9, 1983, p. 1869. http://dx.doi.org/10.1088/0305-4470/16/9/012

[31] L. Nottale, "Fractal Space-Time and Microphysics," World Scientific, Singapore, 1993.

[32] M. S. El Naschie, "Quantum Mechanics, Cantorian Space-Time and the Heisenberg Uncertainty Principle," Vistas in Astronomy, Vol. 37, 1993, pp. 249-252. http://dx.doi.org/10.1016/0083-6656(93)90040-Q

[33] A. Connes, “Non-commutative Geometry,” Academic Press, San Diego, 1994.

[34] M. S. El Naschie, "Penrose Universe and Cantorian Space-Time as a Model for Non-Commutative Quantum Geometry," Chaos, Solitons \& Fractals, Vol. 9, No. 6, 1998, pp. 931-933. http://dx.doi.org/10.1016/S0960-0779(98)00077-0

[35] M. S. El Naschie, "Quantum Gravity Unification via Transfinite Arithmetic and Geometrical Averaging," Chaos, Solitons \& Fractals, Vol. 35, No. 2, 2008, pp. 252-256. http://dx.doi.org/10.1016/j.chaos.2007.07.019

[36] M. S. El Naschie, "On a Transfinite Symmetry Group with 10 to the Power of 19 Dimensions," Chaos, Solitons \& Fractals, Vol. 36, No. 3, 2008, pp. 539-541. http://dx.doi.org/10.1016/j.chaos.2007.09.006

[37] Y. Tanaka, "The Mass Spectrum of Hadrons and E-Infinity Theory," Chaos, Solitons \& Fractals, Vol. 27, No. 4, 2006, pp. 851-863.

http://dx.doi.org/10.1016/j.chaos.2005.04.080

[38] M. S. El Naschie, "Transfinite Harmonization by Taking the Dissonance Out of the Quantum Field Symphony," Chaos, Solitons \& Fractals, Vol. 36, No. 4, 2008, pp. 781-786. http://dx.doi.org/10.1016/j.chaos.2007.09.018

[39] M. S. El Naschie, “Quantum E-Infinity Field Theoretical Derivation of Newton's Gravitational Constant,” International Journal of Nonlinear Sciences and Numerical Simulation, Vol. 8, No. 4, 2007, pp. 469-474.

[40] M. S. El Naschie, "From E-Eight to E-Infinity," Chaos, Solitons \& Fractals, Vol. 35, No. 2, 2008, pp. 285-290. http://dx.doi.org/10.1016/j.chaos.2007.06.111

[41] M. S. El Naschie, "Higgs Mechanism, Quarks Confinement and Black Holes as a Cantorian Space-Time Phase Transition Scenario,” Chaos, Solitons \& Fractals, Vol. 41, No. 2, 2009, pp. 869-874.

http://dx.doi.org/10.1016/j.chaos.2008.04.013

[42] M. S. El Naschie, “On Phase Transition to Quarks Confinement," Chaos, Solitons \& Fractals, Vol. 38, No. 2, 2008, pp. 332-333. http://dx.doi.org/10.1016/j.chaos.2008.03.003

[43] M. S. El Naschie, “On Quarks Confinement and Asymptotic Freedom,” Chaos, Solitons \& Fractals, Vol. 37, No. 5, 2008, pp. 1289-1291. http://dx.doi.org/10.1016/j.chaos.2008.02.002

[44] M. S. El Naschie, "The Internal Dynamics of the Exceptional Lie Symmetry Groups Hierarchy and the Coupling 
Constants of Unification," Chaos, Solitons \& Fractals, Vol. 38, No. 4, 2008, pp. 1031-1038.

http://dx.doi.org/10.1016/j.chaos.2008.04.028

[45] M. S. El Naschie, "The Exceptional Lie Symmetry Groups Hierarchy and the Expected Number of Higgs Bosons," Chaos, Solitons \& Fractals, Vol. 35, No. 2, 2008, pp. 268-273. http://dx.doi.org/10.1016/j.chaos.2007.07.036

[46] M. S. El Naschie, “On D. Gross’ Criticism of S. Eddington and an Exact Calculation of $\bar{\alpha}_{0} \cong 137$," Chaos, Solitons \& Fractals, Vol. 32, No. 4, 2007, pp. 1245-1249. http://dx.doi.org/10.1016/j.chaos.2006.10.035

[47] M. S. El Naschie, "Rigorous Derivation of the Inverse Electromagnetic Fine Structure Constant $\bar{\alpha}=1 / 137.036$ Using Super String Theory and the Holographic Boundary of E-Infinity," Chaos, Solitons \& Fractals, Vol. 32, No. 3, 2007, pp. 893-895. http://dx.doi.org/10.1016/j.chaos.2006.09.055

[48] I. Affleck, "Golden Ratio Seen in a Magnet," Nature, Vol. 464, No. 18, 2010, pp. 362-363. http://dx.doi.org/10.1038/464362a

[49] M. S. El Naschie, "Von Neumann Geometry and E-Infinity Quantum Spacetime,” Chaos, Solitons \& Fractals, Vol. 9, No. 12, 1998, pp. 2023-2030.

[50] M. S. El Naschie, "Arguments for the Compactness and Multiple Connectivity of Our Cosmic Spacetime,” Chaos, Solitons \& Fractals, Vol. 41, No. 5, 2009, pp. 2787-2789. http://dx.doi.org/10.1016/j.chaos.2008.10.011

[51] M. S. El Naschie, "Banach-Tarski Theorem and Cantorian Micro Spacetime,” Chaos, Solitons \& Fractals, Vol. 5, No. 8, 1995, pp. 1503-1508. http://dx.doi.org/10.1016/0960-0779(95)00052-6

[52] M. S. El Naschie, "A Review of Applications and Results of E-Infinity Theory," International Journal of Nonlinear Sciences and Numerical Simulation, Vol. 8, No. 1, 2007, pp. 11-20.

[53] M. S. El Naschie, “The Quantum Gravity Immirzi Parameter-A General Physical and Topological Interpretation," Gravitation and Cosmology, Vol. 19, No. 3, 2013, pp. 151-155. http://dx.doi.org/10.1134/S0202289313030031

[54] M. S. El Naschie, "A Resolution of the Cosmic Dark Energy via a Quantum Entanglement Relativity Theory,” Journal of Quantum Information Science, Vol. 3, No. 1, 2013, pp. 23-26.

http://dx.doi.org/10.4236/jqis.2013.31006

[55] M. S. El Naschie, “Topological-Geometrical and Physical Interpretation of the Dark Energy of the Cosmos as a 'Halo' Energy of the Schrödinger Quantum Wave,” Journal of Modern Physics, Vol. 4, No. 5, 2013, pp. 591-596. http://dx.doi.org/10.4236/jmp.2013.45084

[56] M. S. El Naschie, “The Missing Dark Energy of the Cosmos from Light Cone Topological Velocity and Scaling the Planck Scale,” Open Journal of Microphysics, Vol. 3, No. 3, 2013, pp. 64-70. http://dx.doi.org/10.4236/ojm.2013.33012

[57] M. S. El Naschie, "What Is the Missing Dark Energy in a Nutshell and the Hawking-Hartle Quantum Wave Collapse," International Journal of Astronomy and Astrophysics,
Vol. 3, No. 3, 2013, pp. 205-211. http://dx.doi.org/10.4236/ijaa.2013.33024

[58] M. S. El Naschie, “A Unified Newtonian-Relativistic Quantum Resolution of the Supposedly Missing Dark Energy of the Cosmos and the Constancy of the Speed of Light," International Journal of Modern Nonlinear Theory and Application, Vol. 2, No. 1, 2013, pp. 55-59.

[59] L. Marek-Crnjac, "Modification of Einstein's E $=\mathrm{mc}^{2}$ to $\mathrm{E}=\mathrm{mc}^{2} / 22$," American Journal of Modern Physics, Vol. 2, No. 5, 2013, pp. 255-263.

[60] M. S. El Naschie, "The Quantum Entanglement behind the Missing Dark Energy," Journal of Modern Physics and Applications, Vol. 2, No. 1, 2013, pp. 88-96.

[61] M. S. El Naschie, "Dark Energy from Kaluza-Klein Spacetime and Noether's Theorem via Lagrangian Multiplier Method," Journal of Modern Physics, Vol. 4, No. 6, 2013, pp. 757-760.

http://dx.doi.org/10.4236/jmp.2013.46103

[62] J.-H. He, "Special Issue on Recent Developments on Dark Energy and Dark Matter,” Fractal Space-Time and Non-commutative Geometry in Quantum and High Energy Physics, Vol. 3, No. 1, 2013.

[63] M. S. El Naschie and A. Helal, "Dark Energy Explained via the Hawking-Hartle Quantum Wave and the Topology of Cosmic Crystallography," International Journal of Astronomy and Astrophysics, Vol. 3, No. 3, 2013, pp. 318343. http://dx.doi.org/10.4236/ijaa.2013.33037

[64] M. S. El Naschie, "Quantum Entanglement as a Consequence of a Cantorian Micro Spacetime Geometry," Journal of Quantum Information Science, Vol. 1, No. 2, 2011, pp. 50-53. http://dx.doi.org/10.4236/jqis.2011.12007

[65] M. S. El Naschie, "Chaotic Fractals at the Root of Relativistic Quantum Physics and Cosmology,” International Journal of Modern Nonlinear Theory and Application, Vol. 2, No. 1A, 2013, pp. 78-88.

[66] M. S. El Naschie, “Quantum Entanglement: Where Dark Energy and Negative Gravity Plus Accelerated Expansion of the Universe Comes from," Journal of Quantum Information Science, Vol. 3, No. 2, 2013, pp. 57-77. http://dx.doi.org/10.4236/jqis.2013.32011

[67] M. S. El Naschie, "The Hydrogen Atom Fractal Spectra, the Missing Dark Energy of the Cosmos and Their Hardy Quantum Entanglement,” International Journal of Modern Nonlinear Theory and Application, Vol. 2, No. 3, 2013, pp. 167-169.

http://dx.doi.org/10.4236/ijmnta.2013.23023

[68] M. S. El Naschie, “A Fractal Menger Sponge Spacetime Proposal to Reconcile Measurements and Theoretical Predictions of Cosmic Dark Energy," International Journal of Modern Nonlinear Theory and Application, Vol. 2, No. 2, 2013, pp. 107-121. http://dx.doi.org/10.4236/ijmnta.2013.22014

[69] M. Krizek and L. Somer, “Antigravity_Its Manifestation and Origin," International Journal of Astronomy and Astrophysics, Vol. 3, No. 3, 2013, pp. 227-235. http://dx.doi.org/10.4236/ijaa.2013.33027

[70] C. Calcagni, J. Magueijo and D. Fernandez, "Varying Elec- 
tric Charges in Multi-Scale Spacetimes,” 2013.

[71] D. Finkelstein, "Quantum Sets and Clifford Algebras," International Journal of Theoretical Physics, Vol. 21, No. 6-7, 1982, pp. 489-503. http://dx.doi.org/10.1007/BF02650180

[72] L. H. Kauffman, "Virtual Logic,” Systems Research, Vol. 13, No. 3, 1996, pp. 293-310. http://dx.doi.org/10.1002/(SICI)1099-1735(199609)13:3< 293::AID-SRES99>3.0.CO;2-D

[73] M. Pusey, J. Barrett and T. Randolph, "On the Reality of Quantum State,” Nature Physics, Vol. 8, 2012, pp. 475478.

[74] M. S. El Naschie, "On Twisters in Cantorian E-Infinity Space,” Chaos, Solitons \& Fractals, Vol. 12, No. 4, 2011, pp. 741-746. http://dx.doi.org/10.1016/S0960-0779(00)00193-4

[75] M. S. El Naschie, "On the Uncertainty of Cantorian Geometry and the Two-Slit Experiment," Chaos, Solitons \& Fractals, Vol. 9, No. 3, 1998. pp. 517-529. http://dx.doi.org/10.1016/S0960-0779(97)00150-1

[76] M. S. El Naschie, "Fractal Black Holes and Information," Chaos, Solitons \& Fractals, Vol. 29, No. 1, 2006, pp. 23-35. http://dx.doi.org/10.1016/j.chaos.2005.11.079

[77] M. S. El Naschie, "Wild Topology, Hyperbolic Geometry and Fusion Algebra of High Energy Particle Physics," Chaos, Solitons \& Fractals, Vol. 13, No. 9, 2002, pp. 1935-1945. http://dx.doi.org/10.1016/S0960-0779(01)00242-9

[78] M. S. El Naschie, "Quantum Loops, Wild Topology and Fat Cantor Sets in Transfinite High Energy Physics," Chaos, Solitons \& Fractals, Vol. 13, No. 5, 2002, pp. 1167-1174. http://dx.doi.org/10.1016/S0960-0779(01)00210-7

[79] M. S. El Naschie, “Average Symmetry, Stability and Er- godicity of Multidimensional Cantor Sets,” II Nuovo Cimento B Series 11, Vol. 109, No. 2, 1994, pp. 149-157. http://dx.doi.org/10.1007/BF02727425

[80] M. S. El Naschie, "The VAK of Vacuum Fluctuation, Spontaneous Self-Organization and Complexity Theory Interpretation of High Energy Particle Physics and the Mass Spectrum,” Chaos, Solitons \& Fractals, Vol. 18, No. 2, 2003, pp. 401-420. http://dx.doi.org/10.1016/S0960-0779(03)00098-5

[81] L. Marek-Crnjac, G. Iovane, S. I. Nada and T. Zhong, "The Mathematical Theory of Finite and Infinite Dimensional Topological Spaces and Its Relevance to quantum gravity," Chaos, Solitons \& Fractals, Vol. 42, No. 4, 2009, pp. 1974-1979. http://dx.doi.org/10.1016/j.chaos.2009.03.142

[82] M. S. El Naschie, "The Feynman Path Integral and E-Infinity from Two-Slit Gedanken Experiment," International Journal of Nonlinear Science \& Numerical Simulation, Vol. 6, No. 4, 2005, pp. 335-342.

[83] M. S. El Naschie, "Mohamed El Naschie Answers a Few Questions about this Month's Emerging Research Front in the Field of Physics,” Thomason Essential Science Indicators.

http://esi-topics.com/erf/2004/october04-MohamedElNas chie.html

[84] M. S. El Naschie, "Revising Einstein's E = mc²: A Theoretical Resolution of the Mystery of Dark Energy," Conference Program and Abstracts of The Fourth Arab Int. Conference in Physics \& Material Science, Bibliotheca Alexandrina, Alexandria, October 2012, pp. 1-3.

[85] M. S. El Naschie, “Nash Embedding of Witten’s M-Theory and the Hawking-Hartle Quantum Wave of Dark Energy," Journal of Modern Physics, Vol. 4, No. 10, 2013, pp. 1417-1428. http://dx.doi.org/10.4236/jmp.2013.410170 